\title{
The Research on the Application of AGV System in Logistics Sorting Operation
}

\author{
Yuan Ruiping, Dong Tingting, Li Juntao \\ School of Information, Beijing Wuzi University, Beijing, China
}

Email address:

yuanruiping@bwu.edu.cn (Yuan Ruiping)

\section{To cite this article:}

Yuan Ruiping, Dong Tingting, Li Juntao. The Research on the Application of AGV System in Logistics Sorting Operation. Automation, Control and Intelligent Systems. Vol. 4, No. 5, 2016, pp. 80-83. doi: 10.11648/j.acis.20160405.12

Received: October 11, 2016; Accepted: October 18, 2016; Published: November 7, 2016

\begin{abstract}
With the rapid development of express industry, traditional sorting operation mode has been unable to meet customers' requirements for speed. As modern automatic logistics equipment, Automated Guided Vehicle (AGV) has many advantages. It can not only reduce the number of operators and greatly improve the working productivity, but also has the characters of flexibility and reliability. In this paper, an AGV system used in express sorting operation is built. Then its business process, system architecture and task schedule algorithm are specified. Finally, AGV sorting system is developed using $\mathrm{C \#}$ and simulation experiments are carried out. The efficiency and error rate of AGV sorting system is compared with traditional sorting methods. Simulation results show that it can be more effective and stable to use the AGV system in logistics sorting operation.
\end{abstract}

Keywords: AGV System, Logistics Sorting Operation, Express Delivery

\section{Introduction}

With the development of e-commerce, its scale is increasing rapidly and tends to develop in the direction of "muti-varieties and small batch" because of personalized needs of people. So it promotes the rapid development of the express industry and the industry also plays an increasingly important role in the country's economy and people's daily life. At the same time, there are many problems in the development of e-commerce, such as high logistics distribution cost, long logistics distribution time, etc. Particularly the concentrated outburst of orders requires improving the capacity of logistic distribution.

The velocity of logistics, as one of the important aspects of service quality evaluation in the express industry, is immediately influenced by the efficiency of sorting operation [1-2]. Sorting operation is a process that according to consumers' order requirements or delivery plans, goods are picked out quickly and accurately as far as possible so that they can be classified and processed intensively. Sorting operation cost accounts for about $90 \%$ of the good handling cost in distribution center. However, in the labor-intensive distribution center, people engaged in sorting operation account for $50 \%$ of the total people in distribution and the time spent on sorting operation accounts for $30 \%-40 \%$ of the total working time in distribution center [3]. For sorting operation modes, traditional manual mode has the disadvantages of low efficiency and high error rate [4]. Modern modes such as automated sorting machine and stereoscopic warehouse, which mainly depend on transmission equipment and logistics information technology, have the advantage of higher sorting efficiency, but also have some shortages such as large area occupied, poor flexibility and robustness, etc [5-7].

Automatic Guided Vehicle(AGV) is a kind of advanced automatic logistics equipment which can meet the requirements of sorting operation and turn the "people to goods" sorting mode into "goods to people" sorting mode. At present, AGV is mainly applied in some industries including photo electricity, tobacco, car, bank, printing, etc. In these traditional industries, the application of AGV shows some characteristics of working independently, fixed track and small scale [8-9]. AGV system is that many AGVs work with each other to complete the assigned tasks and eventually to realize the maximization of overall benefits. So AGV system with the characteristics of automation, flexible routes, strong robustness and great flexibility, can well meet the requirements of sorting operation in large scale. 
In this paper, AGV system is applied to logistics sorting operation of goods in regular shape and large scale. Some relevant problems including warehouse layout, system architecture, task allocation, collision avoidance path planning of AGV are studied. Using the distributed communication mode, sorting operation is completed and the maximum benefit of the system is realized. At last, the efficiency of AGV system in sorting operation is analyzed on $\mathrm{c} \#$ experiment simulation platform.

\section{The Business Process of AGV Sorting System}

The number of tasks (indivisible task unit), sorting stations, AGVs and bins is assumed to be $m, n, s$ and $h$ respectively. Task units reach randomly and different bins represent different districts. According to the surplus number of tasks, $m$ tasks are distributed to $n$ sorting stations uniformly. AGVs at pausing area are distributed to $n$ sorting stations. One AGV can only complete one task once and one task can be only completed by one AGV once. At the sorting station, the task is identified automatically and the location of the corresponding bin is sent to AGV, so the goods can be sent to the assigned location of bin by AGV. Then after completing the task, the AGV goes back to the assigned sorting station for the next task according to the regulation of task distribution until all the tasks are completed. At last AGV returns to the pausing area or is automatically powered by charging piles. The process of sorting operation is shown in figure 1.
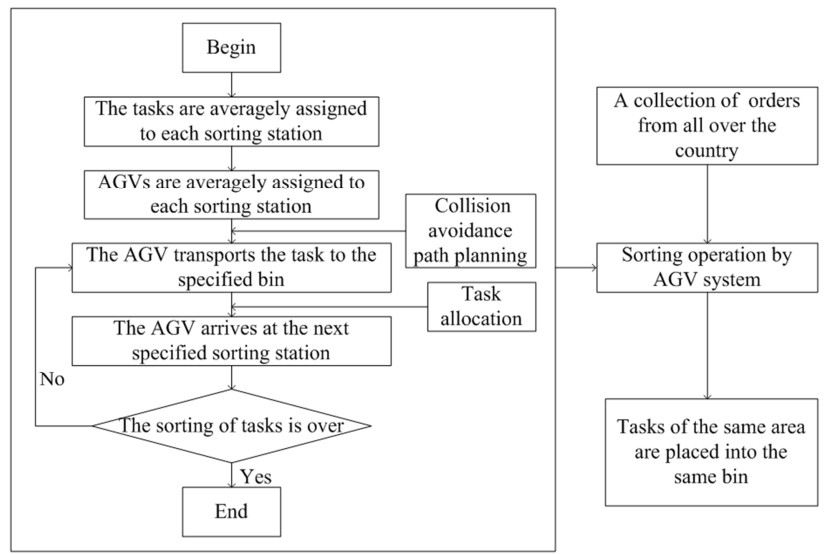

Figure 1. The business process chart of AGV system in sorting operation.

The environmental map model is established using grid method. Grid map modeling is that the environmental map is divided into many grids with the same size which store the information of AGVs' surrounding work environment through two-dimensional network pattern and then the information is translated to the machine languages which the AGV system can understand. In the grid map modeling, the size and number of grids are determined by the size of AGV and work space. The storage environment modeled by grid method in this paper is shown as in figure 2 .

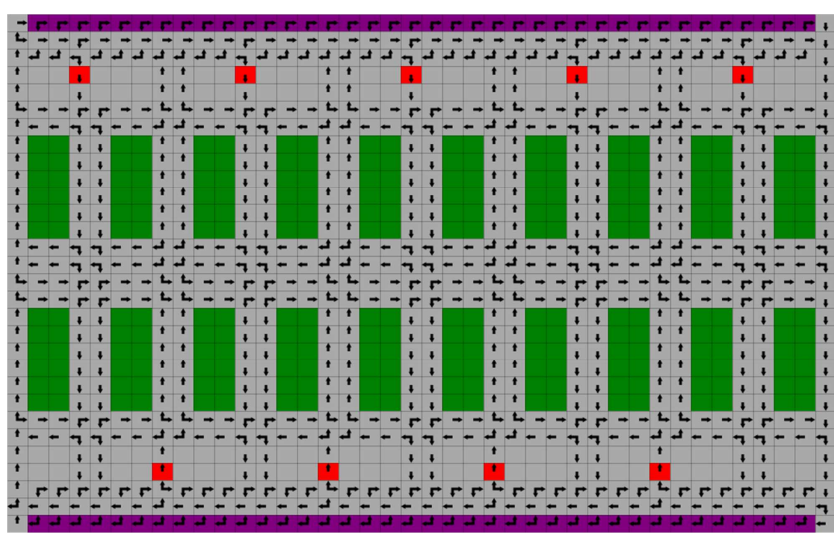

Figure 2. The storage environment modeled by grid method.

In figure 2, there are static obstacles on the surface including sorting station, bin and pausing area, which are represented by red, green and purple grids. The direction of the arrow is AGV's driving direction. AGV enters sorting station from pausing area and waits for being assigned a task which will be placed at the corresponding bin. Then AGV will reach the next assigned sorting stations waiting for another task. When the tasks at all of the sorting stations are finished, the program finishes.

\section{The Architecture of AGV Sorting System}

The distributed control mode is adopted in the AGV sorting system. The functions of processing returned data, assigning tasks and handling errors are undertaken by central control unit. Every AGV has its own processor, so it can calculate its own benefit value and makes real time communication with central control unit through wireless network to send its status information, task fulfillment status and benefit function. Then the central control unit will distribute AGV to the sorting station with the maximum overall benefit to execute next task according to the number of the tasks completed, the number of remaining tasks, the location of $\mathrm{AGV}$, etc. The architecture diagram of sorting operation in AGV system is shown in figure 3 .

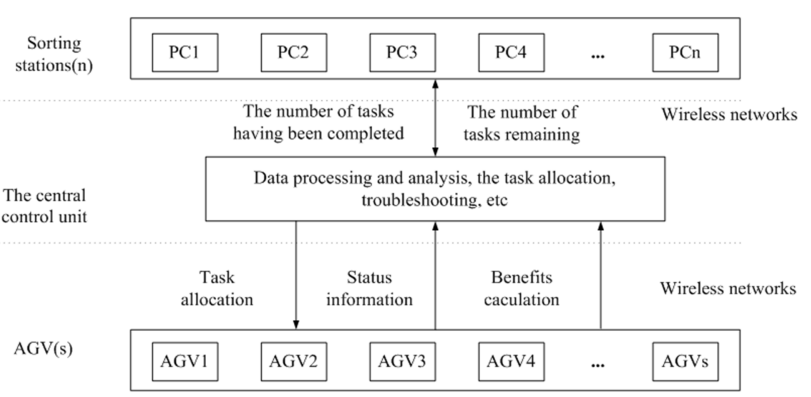

Figure 3. The architecture diagram of $A G V$ system in sorting operations.

The AGV sorting system established in this paper can improve the efficiency of sorting tasks by means of parallel work of many sorting stations, so the system is applicable for 
the express tasks with the characteristic of large batches.

\section{The Task Scheduling of AGV Sorting System}

Task scheduling is the core of sorting operation. Task scheduling mainly includes task allocation, path planning, obstacle avoidance planning and emergency treatment.

\subsection{Task Allocation}

When AGV finishes the current task, it will choose the next sorting station to execute a new task. So in this process, the distribution regulations are involved. Mainly two factors including the efficiency of AGV and the work efficiency of the workers at the sorting station are considered to realize man-machine coordination and the maximization of overall benefits. The efficiency of $\mathrm{AGV}$ is measured by the real distance between $\mathrm{AGV}$ and sorting station. The work efficiency of the workers depends on the number of the tasks completed and the number of remaining tasks.

\subsection{Path Planning}

After the tasks are allocated, when the starting point and the end point are determined, the shortest path is planned by $\mathrm{A}^{*}$ algorithm and the objective function is modified based on the concept of time window to realize dynamic path planning. There are two factors considered including the distance between the current point and the target point and the number of AGVs on the feasible paths. At the intersection, the paths of the AGVs are planned again and all the feasible paths are detected. The penalty value of the paths that AGVs share with each other is added. The penalty value depends on the distance from AGVs.

\subsection{Obstacle Avoidance Planning}

In multiple AGV system, the conflicts between AGVs are handled based on the predetermined priority combined with path planning. Priority rules of AGVs are given as follows: (1)status(loading) $>$ status(no-load);(2)the time spent on the tasks; (3) when two AGVs move in the same direction, the following one must choose waiting strategy; when the AGV moves in the direction which the other does not move in, the AGV with lower priority should wait.

By studying task allocation, path planning and obstacle avoidance planning, the total time spent on finishing all the tasks is decreased and the maximization of benefits is realized eventually.

\section{System Simulation}

On C\# software platform, AGV sorting system is develop, in which task allocation algorithm, path planning algorithm and collision avoidance algorithm of AGV system is integrated. The software interface of the simulation system is shown in Figure 4. Firstly, the menu bar on the left can monitor the running state of $\mathrm{AGV}$ and adjust the speed and acceleration of AGV. Secondly, the work area on the right can simulate the real sorting environment. Lastly, the system can set up the necessary application environment and the task load. Different colors can be set to AGV according to AGV's current states (pausing, turning, loading and no-load). AGV can be analyzed further and its fault can be detected according to different colors.

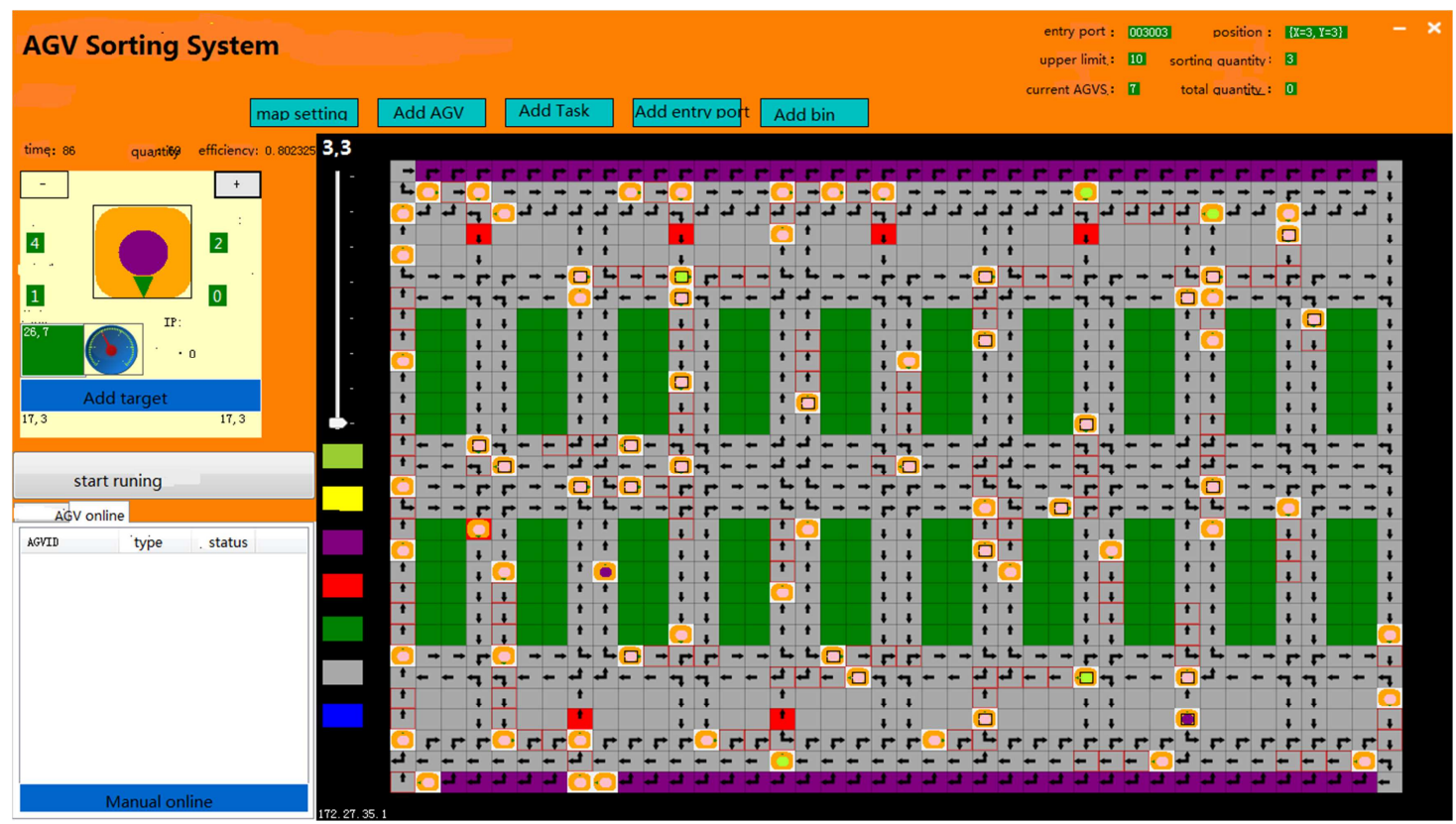

Figure 4. The simulation interface of $A G V$ sorting system.

In this paper, there are 120 AGVs with the speed of 2 meters per second and 8 sorting stations. The simulation results are shown in Table1.we can see that the average sorting efficiency is over 3 pieces per second, which shows that AGV system has 
high sorting efficiency. Although the efficiency of sorting is slightly decreased with the increase of operation time, this is caused by traffic congestion, which can be overcome by improving task scheduling algorithm.

Table 1. Efficiency analysis of the AGV sorting system.

\begin{tabular}{lllll}
\hline $\begin{array}{l}\text { Experiment } \\
\text { number }\end{array}$ & $\begin{array}{l}\text { The number of } \\
\text { AGVs }\end{array}$ & $\begin{array}{l}\text { The number of sorting } \\
\text { stations }\end{array}$ & The number of tasks & $\begin{array}{l}\text { Efficiency } \\
\text { (pieces per second) }\end{array}$ \\
\hline 1 & 120 & 8 & 35856 & 3.32 \\
2 & 120 & 8 & 5616 & 5 \\
3 & 120 & 8 & 77616 & 7 \\
4 & 120 & 8 & 99468 & 3.12 \\
\hline
\end{tabular}

The comparison between the AGV sorting system and other sorting methods, including carbel sorting and manual sorting, is shown in Table 2.

Table 2. The comparison between the AGV system and other sorting methods.

\begin{tabular}{llll}
\hline & The AGV system & Carbel Sorting & Manual sorting \\
\hline $\begin{array}{l}\text { Efficiency } \\
\text { (pieces per second) }\end{array}$ & 3.15 & 3.02 & 0.04 \\
Error rate & $0.8 \%$ & $1 \%$ & $3 \%$ \\
\hline
\end{tabular}

The efficiency value and error rate in table 2 shows that it is more efficient and reliable to use AGV system in sorting operation. In terms of sorting efficiency, the AGV system is similar with the automatic Carbel sorting, but it is obvious that the AGV system has more advantages in cost and flexibility.

\section{Conclusions}

In this paper, an AGV system used in logistics express sorting operation is studied. The business process design, system architecture and task scheduling of the system is specified. Simulation shows that it is more efficient and reliable to use AGV system in sorting operation. In future work, we will focus on the algorithm research of task scheduling to improve the system's efficiency.

\section{Acknowledgement}

This paper is supported by Funding Project for Beijing Intelligent Logistics System Collaborative Innovation Center ; Funding Project for Beijing key laboratory of intelligent logistics system; Science and technology plan general program of Beijing Municipal Education Commission (ID:KM201510037001); Funding Project for Technology Key Project of Municipal Education Commission of Beijing (ID:TSJHG201310037036); The authors also gratefully acknowledge the helpful comments and suggestions of the reviewers, which have improved the presentation.

\section{References}

[1] Tang Niannian. The Research on Sorting Operation of Express Company [D]. Dalian Maritime University, 2012.

[2] Gao Xiaoqian. The Analysis on Sorting Operation of Express Company [J]. Marked Modernization Magazine, 2014(29): $55-57$.
[3] Xiang Wei, Li Ping. A Preliminary Study of the Existing Domestic Express Industry Sorting Technology [J]. China Business (Economic Theory Research), 2013(3): 39-40.

[4] Wang Yanyan, Wu Yaohua, Liu Peng. Sorting Task Optimization of Automatic Sorting System [J]. Journal of Mechanical Engineering, 2011, 47(20): 10-17.

[5] Zhang Y, Wu Y. Research of Efficiency Optimization in Tobacco Automated Sorting System[C].IEEE International Conference on Automation and Logistics. IEEE, 2007: 1209-1213.

[6] Zhang Yigong, Wu Yaohua. The Order Arrangement Optimization of an Automated Sorting System with the Ability of Order Accumulation [J]. Journal of Shandong University (English Science), 2008, 38(5): 67-71.

[7] Zhang Ximei. Picking Operation Optimization and Algorithm Research Based on KIVA System [D]. Beijing University of Posts and Telecommunications, 2015.

[8] Chen Ming, Ni Xiongjun, Zhang Jun, etc. The Application Research on AGV Technology in the Tobacco Industry Logistics System [J]. Logistics \& Material Handling, 2015, 20(10): 170-173.

[9] Sun Yu, Zhang Qian, Jiang Yiding. The Application of AGV Control System in the Tobacco Logistics Process [J]. Automation and Instrumentation, 2012, 27(5): 39-41.

[10] Giuseppe Confessore, Marcello Fabiano, Giacomo Liotta. A Network Flow Based Heuristic Approach for Optimising AGV Movements. Journal of Intelligent Manufacturing, 2013, 24(2): 405-419.

[11] Toshiyuki Miyamoto, Kensuke Inoue. Local and Random Searches for Dispatch and Conflict-free Routing Problem of Capacitated AGV Systems. Computers \& Industrial Engineering, 2016, 91(1): 1-9.

[12] Mohammad Saidi-Mehrabada, Saeed Dehnavi-Arania, etc. An Ant Colony Algorithm (ACA) for Solving the New Integrated Model of Job Shop Scheduling and Conflict-free Routing of AGVs. Computers \& Industrial Engineering, 2015, 86: 2-13. 